\title{
电场强度对等离激元诱导热电子的影响
}

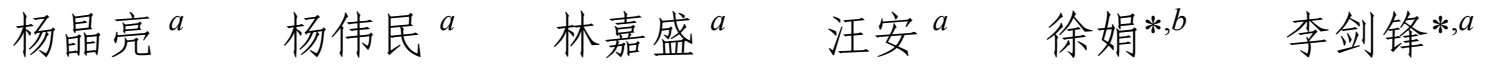 \\ $\left({ }^{a}\right.$ 厦门大学 物理科学与技术学院 化学化工学院 厦门 361005) \\ $\left({ }^{b}\right.$ 闽南师范大学 化学化工与环境学院 漳州 363000)
}

\begin{abstract}
摘要 等离激元纳米结构因其通过改变纳米结构的尺寸、形貌和组成成分, 可以在紫外-可见-近红外范围内实现对光 的操控从而提高能量利用率而受到人们的广泛关注. 在光的激发下, 等离激元纳米结构可以产生高能热电子, 并驱动 光化学反应, 但其利用效率较低. 因此, 如何提升热电子的激发效率成为了一个函待解决的关键问题. 本工作制备了 三维壳层隔绝银纳米粒子载金(3D Ag SHINs-Au)超结构, 以对颈基苯胺(pATP)为探针分子, 结合原位表面增强拉曼光 谱技术和三维有限时域差分法研究不同电场强度对等离激元诱导的热电子激发效率的影响. 实验结果显示电场强度越 强, 热电子激发效率越高, pATP 催化速率越快. 此外, 带内跃迁比带间跃迁更有利于热电子的激发. 本研究有助于人们 理解电场强度如何影响热电子的激发效率.
\end{abstract}

关键词 表面增强拉曼光谱; 等离激元; 热电子; 电场强度; 对統基苯胺

\section{Plasmon-induced Hot Electrons Influenced by Electric Field}

Yang, Jing-Liang ${ }^{a} \quad$ Yang, Wei-Min $^{a} \quad$ Lin, Jia-Sheng $^{a} \quad{\text { Wang, } \mathrm{An}^{a} \quad \mathrm{Xu}, \mathrm{Juan}^{*}, b}_{\mathrm{Li}, \text { Jian-Feng*,a }}$
( ${ }^{a}$ College of Physical Science and Technology, College of Chemistry and Chemical Engineering,
Xiamen University, Xiamen 361005)
( ${ }^{b}$ College of Chemistry, Chemical Engineering and Environment, Minnan Normal University, Zhangzhou 363000)

\begin{abstract}
The plasmonic nanostructures have attracted particular attention due to their superior ability to capture and modulate light in ultraviolet-visible and near-infrared range, by changing the size, morphology, and the composition of nanostructures. Especially in plasmon-driven chemical reactions, plasmon-induced hot electrons (HEs) can be transferred from the surface of metal nanostructures to the lowest unoccupied molecular orbital (LUMO) of the adsorbate molecule or the conduction band of the semiconductor to achieve catalytic reaction. Therefore, how to improve the excitation efficiency of HEs has become a key problem to be solved urgently. In this paper, $120 \mathrm{~nm}$ Ag nanoparticles (NPs) were synthesized by seed growth method using $45 \mathrm{~nm} \mathrm{Au}$ as seed. Subsequently, (3-aminopropyl)trimethoxysilane as coupling agent and sodium silicate as the silicon source were used to prepare the shell-isolated Ag NPs with $2 \sim 3 \mathrm{~nm} \mathrm{SiO}_{2}$ shell (Ag SHINs). Finally, Ag SHINs were modified with poly(allylamine hydrochloride), then small $\mathrm{Au}(\mathrm{ca} .15 \mathrm{~nm})$ as satellites were electrostatic self-assembled onto the surface of Ag SHINs to form a 3D Ag SHINs-Au superstructure. Using $p$-aminothiophenol (pATP) as probe molecule, in-situ surface-enhanced Raman spectroscopy (SERS) was employed to real-timely monitor the catalytic reaction processes from pATP to DMAB, using 532, 638, and $785 \mathrm{~nm}$ lasers for excitation, respectively. The results showed that the highest conversion efficiency was achieved when $638 \mathrm{~nm}$ laser was applied. In addition, the reaction rate under 785 $\mathrm{nm}$ excitation was faster than that under exposure to $532 \mathrm{~nm}$ laser. Then, we used three dimensional (3D) finite-difference time-domain (FDTD) to simulate the electric field distribution of 3D Ag SHINs-Au superstructure. The electric field simulation results are consistent with the experimental results. In consequence, the stronger the electric field intensity, the higher the HEs excitation efficiency. On the other hand, the intra-band transitions produce HEs more efficiently than inter-band transitions. Therefore, this study is helpful for understanding how the electric field intensity affect the excitation efficiency of the HEs.

Keywords SERS; plasmonics; hot electrons; electric field intensity; $p$-aminothiophenol
\end{abstract}

\section{1 引言}

在一定波长的光激发下，等离激元纳米结构引起的 局域表面等离激元共振效应能够将光限域在几个纳米 的微小空间范围内, 即纳米间隙或热点处 ${ }^{[1-4]}$. 热点处的
电场强度与入射光相比将获得几个数量级的提升，当分 子刚好处于该范围内时其拉曼光谱信号也将获得极大 增强，即表面增强拉曼光谱(SERS $)^{[5-8]}$. 该技术具有极 高检测灵敏度，甚至可以达到单分子检测水平，目前已

* E-mail: xjzhejiang.2008@163.com; Li@xmu.edu.cn; Tel.: 0592-2186192

Received May 8, 2020; published June 16, 2020.

Project supported by the National Natural Science Foundation of China (Nos. 21925404, 21703180, 21775127).

项目受国家自然科学基金(Nos. 21925404, 21703180, 21775127)资助. 
经被广泛地应用于生物检测 ${ }^{[9]}$ 、原位监测催化反应 ${ }^{[10]}$ 、 环境监测 ${ }^{[11]}$ 等诸多领域. 同时, 处在热点处的分子不仅 受到来自纳米粒子表面的电场增强, 还会因为纳米粒子 表面产生的热电子而驱动光化学反应 ${ }^{[12,13]}$. 因此, 该技 术将表面化学反应与原位拉曼监测结合在一起, 为探究 真实体系下的反应过程提供了一个更加方便快捷的平 台.

通常情况下等离激元驱动的光化学反应是通过等 离激元共振引起的非辐射衰减产生热电子引起 ${ }^{[14-17]}$. 其 衰减过程常经历三个步骤: (1)纳米结构在光激发下诱导 产生等离激元共振效应, 并形成表面电场; (2)等离激元 共振效应在 Landau 阻尼下产生热电子; (3)热电子从纳 米结构表面转移至分子的最低非占据轨道(LUMO)或半 导体的导带上, 最终实现催化反应. 事实上, 纳米结构 表面的电场强度越大其表面的电荷密度越大, 相应的单 位时间内电子与激发光之间的能量交换几率增加, Landau 阻尼更容易发生, 因此, 更容易激发出更多的热电 子. 为了更好地提升热电子的利用效率人们分别合成了 $\mathrm{Ag} @ \mathrm{TiO}_{2}{ }^{[18]}, \mathrm{Au} @ \mathrm{CdS}^{[19]}$ 等结构去研究热电子引起的催 化反应过程. 然而, 在真实催化体系下该类结构往往以 单颗粒形式存在. 其表面的电场强度较弱, 导致热电子 的激发和转移效率低. 因此, 如何更加高效地提升对热 电子的激发和利用效率成为一个亟待解决的关键问题.

本工作中, 以 $15 \mathrm{~nm}$ 小金纳米粒子作为卫星结构组 装在壳厚为 $2 \sim 3 \mathrm{~nm}$ 的超薄 $\mathrm{SiO}_{2}$ 壳层隔绝的银纳米粒 子(Ag SHINs)上形成 3D Ag SHINs-Au 超结构, 同时以 对颈基苯胺(pATP)为探针分子, 采用原位 SERS 技术研 究了 pATP 分子在不同的激光波长下的反应速率. 最终 实验结果表明, 该结构在 $638 \mathrm{~nm}$ 激光激发下具有最佳 的催化转化速率. 随后结合三维有限时域差分法 (3D-FDTD)对超结构体系进行模拟计算发现, 在 $638 \mathrm{~nm}$ 激光下 $3 \mathrm{D} \mathrm{Ag}$ SHINs-Au 结构表面具有最强的电场强度. 这与我们的实验结果完全吻合. 后续的实验数据分析还 发现带内跃迁产生的热电子激发效率远远大于带间跃 迁产生热电子的激发效率. 这为以后更加合理地设计催 化剂提供了一定的指导.

\section{2 结果与讨论}

\section{$2.13 \mathrm{D} \mathrm{Ag} \mathrm{SHINs-Au}$ 的制备及表征}

为了探究不同电场强度对 pATP 分子催化转化速率 的影响, 合成了 $3 \mathrm{D}$ Ag SHINs-Au 超结构纳米粒子. 首 先, 以柠檬酸钠为还原剂分别合成了尺寸为 $15 \mathrm{~nm}$ 和 45 $\mathrm{nm}$ 的金纳米粒子, 随后以 $45 \mathrm{~nm}$ 金纳米粒子为种子合 成了尺寸为 $120 \mathrm{~nm}$ 的银纳米粒子, 以氨丙基三甲氧基 硅烷(APTMS)为偶联剂在其表面包覆上厚度为 $2 \sim 3 \mathrm{~nm}$ 的致密 $\mathrm{SiO}_{2}$ 壳层, 形成壳层隔绝的 $\mathrm{Ag}$ SHINs. 为避免 修饰剂对卫星结构的催化活性造成影响, 将聚丙烯胺盐 酸盐(PAH)修饰在 Ag SHINs 一端, 采用静电自组装的方
式将尺寸为 $15 \mathrm{~nm}$ 的小金纳米粒子组装在 $\mathrm{Ag}$ SHINs 上, 形成单分散的 3D Ag SHINs-Au 超结构水溶液体系. 在 该体系中, 由于惰性壳层 $\mathrm{SiO}_{2}$ 的保护, 内层的 $\mathrm{Ag}$ 核只 提供电场增强作用, 不参与任何催化反应.

其结构示意图以及紫外可见吸收光谱图如图 1a 所 示. 将小 $\mathrm{Au}$ 组装在大的 $\mathrm{Ag}$ SHINs 上后其吸收峰出现了 明显的蓝移现象, 这主要是因为在该结构中 $\mathrm{Ag}$ SHINs 的吸收占主导作用, 且与单纯的 $\mathrm{Au}$ 相比 $\mathrm{Ag}$ SHINs-Au 的吸收强度也有明显增加, 相应的透射电子显微镜 (TEM)和扫描电子显微镜(SEM) 图如图 $1 \mathrm{~b}$ 所示: 大面积 的 SEM 以及 TEM 图片都说明小 $\mathrm{Au}$ 纳米粒子均匀地分 布在大的 $\mathrm{Ag}$ SHINs 表面, 形成了稳定的 3D 超结构. 这 为我们后续研究水溶液环境下单分散体系的实际催化 过程提供了可能.
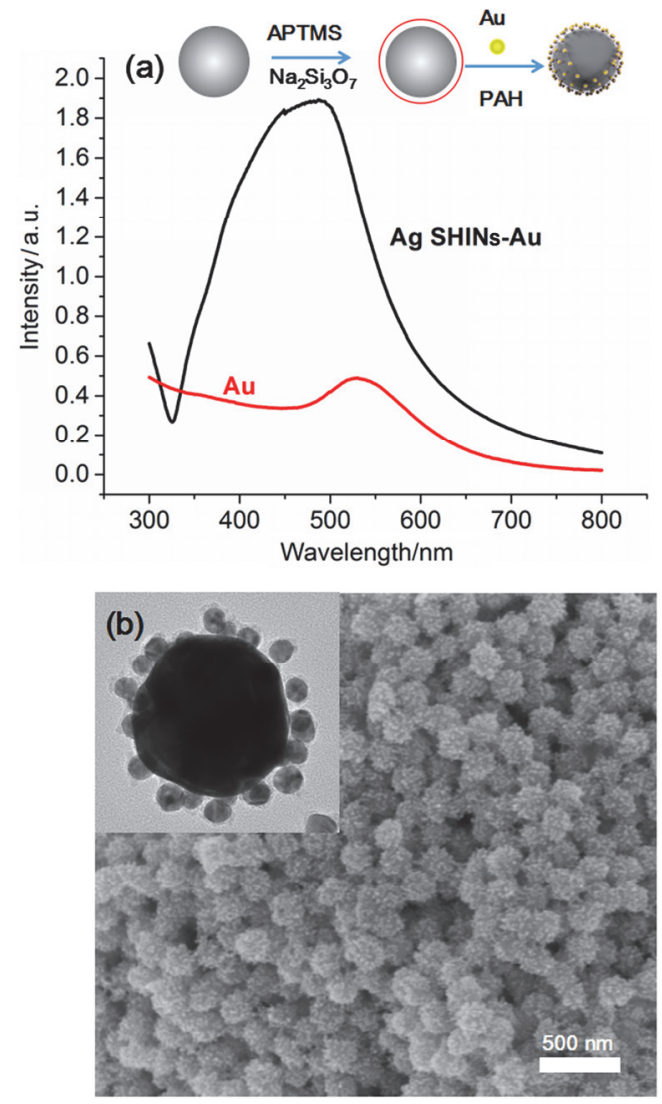

图 1 (a) 3D Ag SHINs-Au 超结构合成过程示意图; (b) 3D Ag SHINs-Au 超结构的 SEM 图以及 TEM 图.

Figure 1 (a) Schematic diagram of 3D Ag SHINs-Au preparation process; (b) TEM (inset) and SEM image of 3D Ag SHINs-Au superstructure.

\section{2 不同电场强度对 pATP 催化速率的影响}

为了研究不同的电场强度对热电子激发效率的影 响, 以 pATP 为探针分子, 将其与水溶液分散的 $3 \mathrm{D} \mathrm{Ag}$ SHINs-Au 超结构混合, 最终保证在该体系中 pATP 分子 浓度维持在 $1 \mathrm{mmol} \cdot \mathrm{L}^{-1}$. 随后分别用 $532,638,785 \mathrm{~nm}$ 激光进行实验. 其实验结果如图 2 所示. 
从图 $2 \mathrm{a}$ 可知, 在 $638 \mathrm{~nm}$ 激光的连续曝光下, 实验 迅速在 1140,1390 和 $1430 \mathrm{~cm}^{-1}$ 处检测到新的拉曼谱峰, 这些新的谱峰对应了 4,4'-二䘪基偶氮苯(DMAB)分子的 $\mathrm{C}-\mathrm{N}$ 键和 $\mathrm{N}=\mathrm{N}$ 键. 这一结果说明, $\mathrm{pATP}$ 在等离激元 诱导的热电子的催化作用下快速转化成 DMAB. 经过 $20 \mathrm{~s}$ 的反应时间后, $1140 \mathrm{~cm}^{-1}$ 处的峰强度逐渐升高, 相 应的 pATP 分子在 $1596 \mathrm{~cm}^{-1}$ 处的 SERS 峰逐渐降低. 这 说明在整个体系中的反应底物 pATP 的量逐渐减少, 相 应的反应产物 DMAB 随之增多, 最终反应速率变缓. 另 外, 从图 $2 \mathrm{~b}$ 和 $2 \mathrm{c}$ 中可知无论在 $785 \mathrm{~nm}$ 还是 $532 \mathrm{~nm}$ 激 发下都有 DMAB 的生成. 然而, 在 $785 \mathrm{~nm}$ 激发下 pATP 分子的转化速率明显快于 $532 \mathrm{~nm}$ 激发. 由于 $785 \mathrm{~nm}$ 激 光激发电子的带内跃迁, 而 $532 \mathrm{~nm}$ 激光激发电子的带 间跃迁, 且 $532 \mathrm{~nm}$ 激光功率略强于 $785 \mathrm{~nm}$ 激光的功率, 这说明在该体系中带内跃迁产生的热电子数目明显多 于带间跃迁产生的热电子数目. 因此证明等离激元诱导 带内跃迁产生热电子的效率高于带间跃迁作用. 另一方 面, 图 2c 中 pATP 分子在 $532 \mathrm{~nm}$ 激发时转化成 DMAB 的 SERS 谱线的基线偏高, 这说明在该激发波长下有很 强的热效应产生. 这是因为在 $532 \mathrm{~nm}$ 激光下 $\mathrm{Au}$ 纳米粒 子处于带间跃迁，因此，热效应明显. 随后实验对比了 该 3D Ag SHINs-Au 超结构分别在 532, 638, $785 \mathrm{~nm}$ 激光 波长下对 pATP 催化转化成 DMAB 的影响. 由于 1080 $\mathrm{cm}^{-1}$ 处的拉曼信号峰归属于 pATP 和 DMAB 分子中 $\mathrm{C}-\mathrm{S}$ 键的伸缩振动, 反应过程中该拉曼峰强基本保持 不变. 因此, 以 $1140 \mathrm{~cm}^{-1}$ 的峰面积与 $1080 \mathrm{~cm}^{-1}$ 峰面积 之间的比值拟合 pATP 的催化转化曲线与曝光时间之间
的关系, 其结果如图 $2 \mathrm{~d}$ 所示. 图中显示, 在 $638 \mathrm{~nm}$ 激光 下 3D Ag SHINs-Au 超结构表现出最佳的催化转化速率.

\subsection{D-FDTD 模拟计算结果}

为了更好理解激发波长对 $\mathrm{pATP}$ 分子催化转化速率 的影响, 以包覆有 $2 \mathrm{~nm} \mathrm{SiO} 2$ 壳层的 $120 \mathrm{~nm} \mathrm{Ag}$ 为内核, 以 $15 \mathrm{~nm} \mathrm{Au}$ 纳米粒子为卫星, 金纳米粒子覆盖度为 $60 \%$, 激光的电场强度选取为 $1 \mathrm{~V} \cdot \mathrm{m}^{-1}$, 偏振方向及传 播方向如图所示, 建立计算模型，结合 3D-FDTD 对 3D Ag SHINs-Au 超结构在水溶液体系下，用 532, 638, 785 $\mathrm{nm}$ 激光激发其表面时的电场分布情况进行模拟计算. 其计算结果如图 3 所示.

从图 3 中可知在不同的激发光条件下 $3 \mathrm{D} \mathrm{Ag}$ SHINs-Au 超结构表面的归一化电场强度分布情况为: $638 \mathrm{~nm}>785 \mathrm{~nm}>532 \mathrm{~nm}$. 模拟结果显示在 $638 \mathrm{~nm}$ 激 发波长下该结构具有最强的电场强度，其最大增强因子 为 $E^{4}=7.6 \times 10^{6}$. 事实上电场强度的大小直接决定了纳 米结构表面的电荷密度的大小, 电场强度越强表面的电 荷密度越大，相应的在单位时间内电子与激发光之间发 现能量交换的几率越大，越容易发生 Landau 阻尼而产 生更多的热电子. 与此同时，由于 $638 \mathrm{~nm}$ 和 $785 \mathrm{~nm}$ 激 光激发的电子都属于带内跃迁, $638 \mathrm{~nm}$ 与 $785 \mathrm{~nm}$ 相比 具有更高的激发能量, 因此 $638 \mathrm{~nm}$ 激光激发获得的热 电子往往具有更高的能量. 相应的由于在 $532 \mathrm{~nm}$ 激发 波长下激发的是电子的带间跃迁, 电子跃迁至费米能级 之上的有效能量较低. 因此, 我们认为在 $638 \mathrm{~nm}$ 激发波 长下无论是产生的热电子数量还是热电子的能量都高
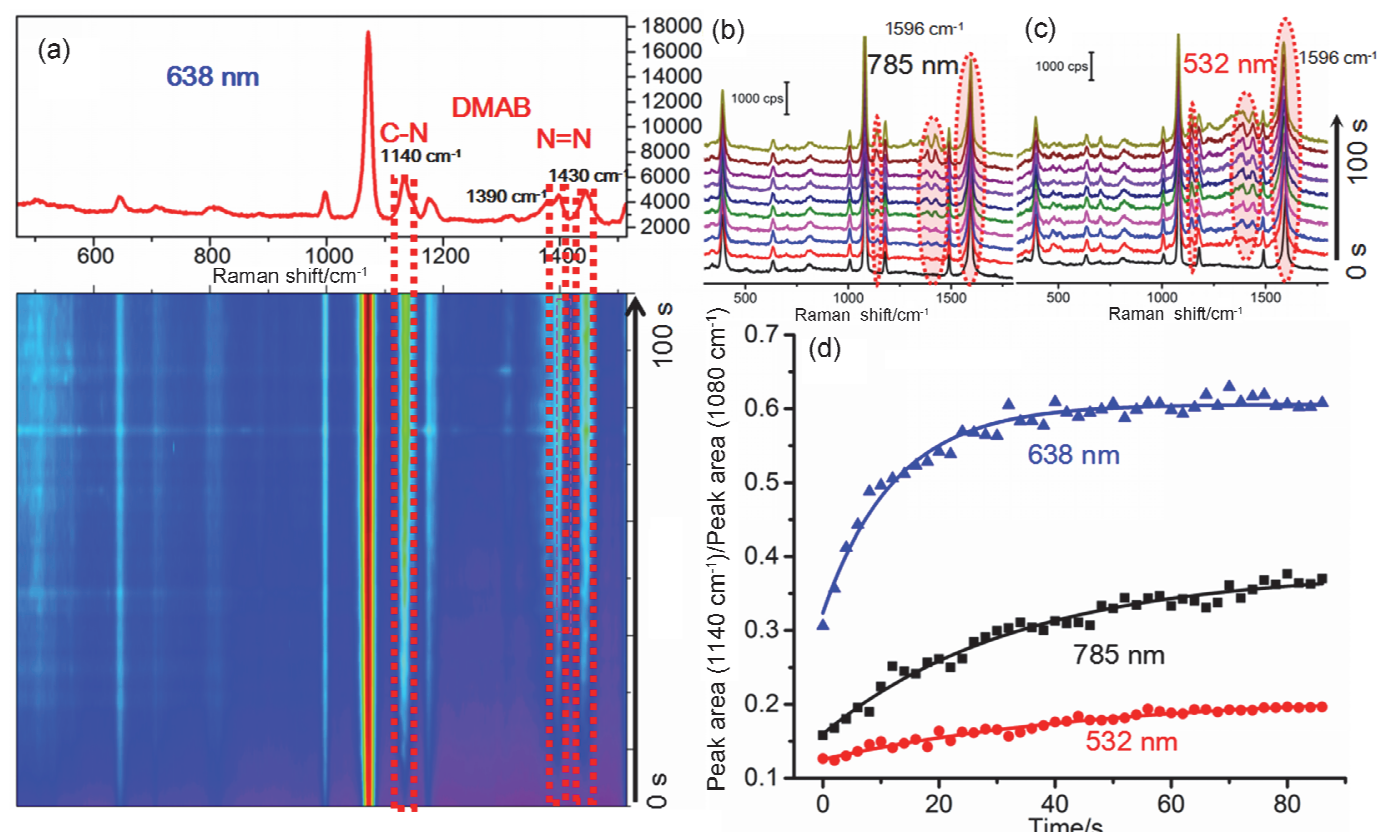

图 2 (a) $638 \mathrm{~nm}$ 激光下 pATP 分子转化为 DMAB 的时间 mapping 图; (b, c) pATP 分子分别在 $785 \mathrm{~nm}$ 和 $532 \mathrm{~nm}$ 激光下随曝光时间转化为 DMAB 的 SERS 光谱图; (d) 在不同激光波长下 pATP 转化为 DMAB 的速率曲线.

Figure 2 (a) Raman mapping for photocatalytic reaction of pATP to DMAB, under $638 \mathrm{~nm}$ laser; (b, c) the SERS spectra of pATP conversion to DMAB under $785 \mathrm{~nm}$ and $532 \mathrm{~nm}$ laser, respectively; (d) rate curves of pATP conversion to DMAB. 

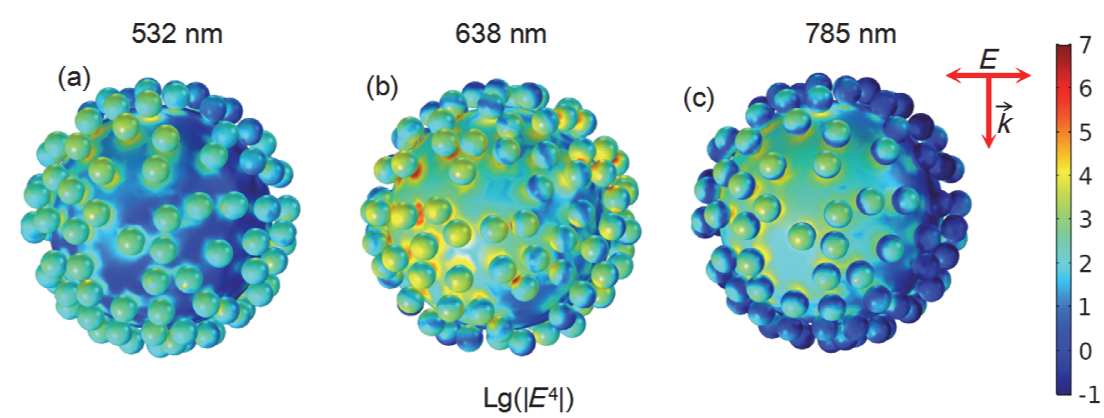

图 3 3D Ag SHINs-Au 超结构在(a) $532 \mathrm{~nm}$, (b) $638 \mathrm{~nm}$ 和(c) $785 \mathrm{~nm}$ 激发波长下的电场分布

Figure 3 Simulated electric field distribution of 3D Ag SHINs-Au superstructure at the (a) $532 \mathrm{~nm}$, (b) $638 \mathrm{~nm}$ and (c) $785 \mathrm{~nm}$ excitation wavelength, respectively.

于 $785 \mathrm{~nm}$ 和 $532 \mathrm{~nm}$. 这一模拟结果很好地解释了为何 在 $638 \mathrm{~nm}$ 激发下 3D Ag SHINs-Au 超结构具有最佳的催 化转化效果. 以上结果表明纳米粒子表面电场强度直接 影响热电子的激发效率, 这为以后更加合理地设计等离 激元诱导的热电子催化剂提供了一定的依据.

\section{4 理论分析结果}

为了更好地分析本项研究中的实验体系, 理解热电 子诱导的催化反应效率与电场强度及电子跃迁的关系, 图 4 中绘制了电子跃迁示意图和催化剂示意图.

金纳米粒子产生带间跃迁所需电子能量约为 2.3 $\mathrm{eV}$. 因此, 当用 $532 \mathrm{~nm}$ 激发光对金纳米粒子进行激发 时, 将使得电子从金的 $5 \mathrm{~d}$ 轨道跃迁至位于费米能级之 上的 $6 \mathrm{sp}$ 轨道, 此时当产生的热电子能量足够跃迁至分 子的 LUMO 轨道时, 即发生热电子的有效转移. 然而, 如图 4a 所示, 带间跃迁是热电子从 $5 \mathrm{~d}$ 轨道至位于费米 能级之上 $6 \mathrm{sp}$ 轨道. 热电子具有的有效能量 $\Delta E$ 较低. 对 于带内跃迁, 如图 $4 \mathrm{~b}$ 所示, 电子从位于费米能级之下的 $6 \mathrm{sp}$ 轨道跃迁至位于费米能级之上的 $6 \mathrm{sp}$ 轨道. 热电子 具有的有效能量 $\Delta E$ 较高. 因此, 与带间跃迁相比, 带内 跃迁更易产生高能热电子, 且相同激光能量下可产生数 目更多的热电子. 另外, 从图 $4 \mathrm{a}$ 和 $4 \mathrm{~b}$ 的对比发现, 带内 跃迁产生的热电子能量普遍高于带间跃迁产生的热电 子能量. 另一方面, 实验结果和理论计算都表明电场强
度越强的位置, 热电子激发效率越高. 结合上述讨论, 如图 $4 \mathrm{c}$ 所示, 在 $638 \mathrm{~nm}$ 激光激发下, 在 $\mathrm{Au}$ 纳米粒子和 $\mathrm{Ag}$ 纳米粒子形成的热点位置能够产生更多的高能热电 子用于催化反应，因而该位点反应速率相对于其它位点 更快.

\section{3 结论}

综上所述, 本研究以 $3 \mathrm{D} \mathrm{Ag} \mathrm{SHINs-Au}$ 超结构为催 化剂研究了 pATP 分子在不同电场强度作用下的催化转 化速率. 实验结果发现在不同的激发波长下 $3 \mathrm{D} \mathrm{Ag}$ SHINs-Au 对 pATP 具有完全不同的催化转化速率, 其中 $638 \mathrm{~nm}$ 激发时催化转化最快. 随后结合 3D-FDTD 对 3D $\mathrm{Ag}$ SHINs-Au 超结构在不同激发波长下的电场分布进 行了详细地模拟计算, 模拟结果显示在 $638 \mathrm{~nm}$ 激发波 长下该结构具有最强的电场强度. 这说明电场强度越强 热电子产生越多, 相应的催化速率越快. 此外, 还发现 $785 \mathrm{~nm}$ 激光激发时其催化转化速率也同样快于 $532 \mathrm{~nm}$ 激发. 我们认为主要是带内跃迁产生的热电子无论是数 目或能量都远远高于带间跃迁，而带间跃迁产生的低能 热电子只有极少部分的热电子能够实现有效转移. 因 此, 带内跃迁产生的热电子更有利于提高催化反应效 率. 以上实验结果为以后更加可靠合理地设计催化剂提 供了一定的依据及指导意义. (a)

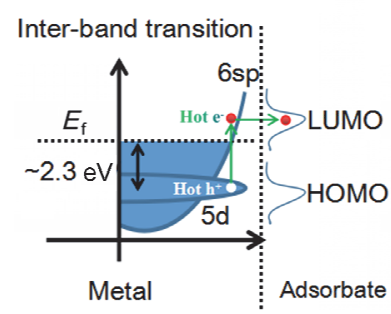

(b)

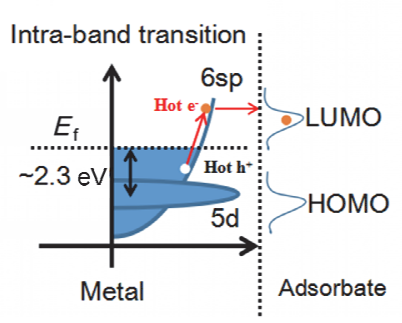

(c)

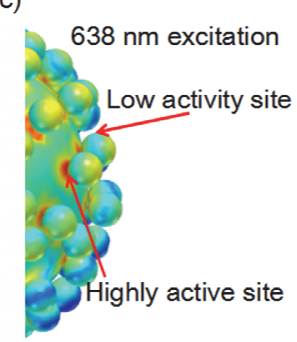

图 4 (a) 带间跃迁下热电子从金属转移至吸附分子的示意图; (b) 带内跃迁下热电子从金属转移至吸附分子的示意图; (c) 不同反应位点下分子 的催化反应速率示意图.

Figure 4 (a) Schematic diagram of hot electrons transfer from metal to adsorbate molecules during inter-band transitions; (b) schematic diagram of hot electrons transfer from metal to adsorbate molecules during intra-band transitions; and (c) schematic diagram of catalytic reaction rates of pATP molecules at different reaction sites. 


\section{4 实验部分}

采用种子生长法合成粒径为 $120 \mathrm{~nm}$ 的 $\mathrm{Ag}$ 纳米粒 子, 具体操作步骤如下: 取 $60 \mathrm{~mL}$ 超纯水, 以 $10 \mathrm{~mL} 45$ $\mathrm{nm}$ 的 $\mathrm{Au}$ 为种子, $0.3 \mathrm{~mL} 1 \mathrm{wt} \%$ 的柠檬酸钠为保护剂, 1.2 $\mathrm{mL} 1 \mathrm{wt} \%$ 的抗坏血酸为还原剂在常温下, 缓慢滴加 10 $\mathrm{mL} 2 \mathrm{mmol} \cdot \mathrm{L}^{-1}$ 的 $\mathrm{AgNO}_{3}, 30 \mathrm{~min}$ 后即可获得尺寸均匀 的 $120 \mathrm{~nm} \mathrm{Ag}$ 纳米粒子. 随后以 $1.2 \mathrm{~mL} 1 \mathrm{mmol} \cdot \mathrm{L}^{-1}$ 的 APTMS 为偶联剂, $3.2 \mathrm{~mL} 0.54 \mathrm{wt} \%$ 的硅酸钠为硅源, 调 节体系 $\mathrm{pH}=10.2$, 随后转移至 $99{ }^{\circ} \mathrm{C}$ 水浴条件下反应 30 $\mathrm{min}$, 即可获得壳厚为 $2 \sim 3 \mathrm{~nm}$ 的壳层隔绝银纳米粒子 Ag SHINs.

以 $\mathrm{PAH}$ 为修饰剂, 取 $10 \mu \mathrm{L} 1 \mathrm{mmol} \cdot \mathrm{L}^{-1}$ 的 $\mathrm{PAH}$ 与 1

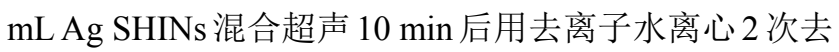
掉多余的 PAH, 将体系体积还原成 $1 \mathrm{~mL}$, 随后采用静电 自组装的方式将 $3 \mathrm{~mL} 15 \mathrm{~nm}$ 的小 $\mathrm{Au}$ 纳米粒子加入到 1 $\mathrm{mL}$ 修饰有 $\mathrm{PAH}$ 的 Ag SHINs 水溶液中, 超声 $10 \mathrm{~min}$ 后 将多余的小 $\mathrm{Au}$ 离心 2 次, 最终形成以 $\mathrm{Au}$ 为卫星的 $3 \mathrm{D}$ $\mathrm{Ag}$ SHINs-Au 超结构. 将其直接与一定浓度的 pATP 水 溶液分子混合, 维持 pATP 分子的最终浓度为 $1 \mathrm{mmol} \cdot$ $\mathrm{L}^{-1}$. 最终, 分别在 532, 638 和 $785 \mathrm{~nm}$ 激光的连续曝光 下测试 pATP 分子转化为 DMAB 的 SERS 光谱. 其中 532,638 和 $785 \mathrm{~nm}$ 激光下的采谱条件均为 $1 \%$ 功率, 连 续曝光 $100 \mathrm{~s}$, 采谱间隔 $2 \mathrm{~s}$.

\section{References}

[1] Moskovits, M. Rev. Mod. Phys. 1985, 57, 783.

[2] Nie, S.; Emory, S. R. Science 1997, 275, 1102

[3] Gersten, J. I.; Birke, R. L.; Lombardi, J. R. Phys. Rev. Lett. 1979, 43 147.

[4] Li, C. Y.; Le, J. B.; Wang, Y. H.; Chen, S.; Yang, Z. L.; Li, J. F.; Cheng, J.; Tian, Z. Q. Nat. Mater. 2019, 18, 697.

[5] Dong, J. C.; Zhang, X. G.; Briega-Martos, V.; Jin, X.; Yang, J.; Chen, S.; Yang, Z. L.; Wu, D. Y.; Feliu, J. M.; Williams, C. T.; Tian, Z. Q.; Li, J. F. Nat. Energy 2019, 4, 60.

[6] Fleischmann, M.; Hendra, P. J.; McQuillan, A. J. Chem. Phys. Lett 1974, 26, 163.

[7] Kozich, V.; Werncke, W. J. Phys. Chem. C 2010, 114, 10484.

[8] Li, J. F.; Huang, Y. F.; Ding, Y.; Yang, Z. L.; Li, S. B.; Zhou, X. S.; Fan, F. R.; Zhang, W.; Zhou, Z. Y.; Wu, D. Y.; Ren, B.; Wang, Z. L.; Tian, Z. Q. Nature 2010, 464, 392.

[9] Gao, Z. G.; Zhen, T. T.; Deng, J.; Li, X. R.; Qu, Y. Y.; Lu, Y.; Liu, T. J.; Luo, Y.; Zhao, W. J.; Lin, B. C. Acta Chim. Sinica 2017, 75, 355 (in Chinese). (高志刚, 郑婷婷, 邓九, 李晓瑞, 曲玥阳, 陆瑶, 刘 婷娇, 罗勇, 赵伟杰, 林炳承, 化学学报, 2017, 75, 355.)

[10] Zhang, H.; Wei, J.; Zhang, X. G.; Zhang, Y. J.; Radjenovica, P. M.; Wu, D. Y.; Pan, F.; Tian, Z. Q.; Li J. F. Chem 2020, 6, 1.

[11] Zuo, F. T.; Xu, W.; Zhao, A. W. Acta Chim. Sinica 2019, 77, 379 (in Chinese). (左方涛, 徐威, 赵爱武, 化学学报, 2019, 77, 379.)

[12] Avanesian, T.; Christopher, P. J. Phys. Chem. C 2014, 48, 28017.

[13] Wei, Q.; Wu, S.; Sun, Y. Adv. Mater. 2018, 48, e1802082.

[14] Besteiro, L. V.; Kong, X. T.; Wang, Z.; Hartland, G.; Govorov, A. O. ACS Photonics 2017, 11, 2759.

[15] Kazuma, E.; Kim, Y. Angew. Chem. Int. Ed. 2019, 15, 4800.

[16] Zhang, J.; Guan, M. X.; Lischner, J.; Meng, S.; Prezhdo, O. V. Nano Lett. 2019, 19, 3187.

[17] Kim, M.; Lin, M.; Son, J.; Xu, H.; Nam, J. M. Adv. Opt. Mater. 2017, 15, 1700004 .

[18] Peckus, D.; Rong, H.; Stankevičius, L.; Juodènas, M.; Tamulevičius, S.; Tamulevičius, T.; Henzie, J. J. Phys. Chem. C 2017, 43, 24159.

[19] Yang, J. L.; Xu, J.; Ren, H.; Sun, L.; Xu, Q. C.; Zhang, H.; Li, J. F.; Tian, Z. Q. Nanoscale 2017, 9, 6254.

(Cheng, B.) 